\title{
PRODUTIVIDADE DO FEIJOEIRO SOB DIFERENTES UNIFORMIDADES DE DISTRIBUIÇÃO DE ÁGUA NA SUPERFÍCIE E NA SUBSUPERFÍCIE DO SOLO
}

\section{JOSÉ A. FRIZZONE ${ }^{1}$, ROBERTO REZENDE ${ }^{2}$, ANTONIO C. A. GONÇALVES ${ }^{2}$, CELSO HELBEL JÚNIOR ${ }^{3}$}

\begin{abstract}
RESUMO: Com o objetivo de comparar a uniformidade de lâminas aplicadas, uniformidade de umidades no solo e produtividade da cultura do feijoeiro, conduziu-se um experimento utilizando a cultivar de feijão IAPAR 57. Foram feitas aplicações de água na superfície do solo, com quatro valores de uniformidades, aplicando-se a mesma lâmina, utilizando-se de quatro espaçamentos entre aspersores: 12 × $12 \mathrm{~m} ; 18 \times 18 \mathrm{~m} ; 18$ × $24 \mathrm{~m}$, e 24 × $24 \mathrm{~m}$. Foram feitas análises das distribuições dos dados de lâmina aplicada, de umidade do solo e de rendimento de grãos, no espaço. Para isso, foram usadas técnicas de análise exploratória que consistiram principalmente em gráficos tipo "box-plot" simplificados, nos quais são apresentados apenas os valores de mediana, máximo e mínimo das distribuições. Verificou-se que a qualidade da irrigação interferiu na uniformidade da umidade do solo e influenciou nas variáveis de produção da cultura de feijão. Uniformidade máxima não implicou maior produtividade de grãos.
\end{abstract}

PALAVRAS-CHAVE: análise exploratória, uniformidade, Phaseolus vulgaris.

\section{DRY BEAN YIELD UNDER DIFFERENT UNIFORMITY OF WATER DISTRIBUTION ON SOIL SURFACE AND UNDERSURFACE}

\begin{abstract}
Aiming to evaluate the effect of irrigation depth and soil moisture uniformity on dry bean yield, an experiment was conducted with the cultivar IAPAR 57. Water was applied on soil surface at four uniformity levels with the same irrigation depth, imposed by four sprinklers spaced at $12 \times 12 \mathrm{~m} ; 18 \times 18 \mathrm{~m} ; 18 \times 24 \mathrm{~m}$, and $24 \times 24 \mathrm{~m}$. Spatial distribution analyses of the irrigation depth, soil moisture and grain yield data were proceeded with exploratory data analysis techniques based on simplified box-plot graphics, which only represent the median, minimal, and maximal values. It was verified that the quality of irrigation affected the soil moisture uniformity, causing effect also on dry bean yield. The maximum uniformity of water distribution did not relate with the highest yield.
\end{abstract}

KEYWORDS: exploratory data analysis, uniformity, Phaseoalus vulgaris.

\section{INTRODUÇÃO}

Diferentes pesquisas têm mostrado a importância da redistribuição de água no solo em relação à distribuição da água na superfície com os equipamentos de irrigação. A aplicação desuniforme de água ao solo cria gradientes de potencial que provocam o movimento da água de pontos de maior potencial para pontos de menor potencial. Há intensa redistribuição dentro do solo e, conseqüentemente, uniformização da distribuição de umidade.

GOHRING \& WALLENDER (1987) e MANTOVANI (1993) afirmaram que a uniformidade de aplicação de água relaciona-se com o rendimento da cultura. Porém, em condições de limitada disponibilidade de água, a uniformidade pode ser compensada por manejo eficiente da lâmina a ser aplicada, proporcionando melhor eficiência na sua utilização.

\footnotetext{
${ }^{1}$ Prof. Dr., Departamento de Engenharia Rural, ESALQ/USP, Piracicaba - SP.

${ }^{2}$ Prof. Dr., Departamento de Agronomia, DAG/UEM, Maringá - PR, rrezende@uem.br

${ }^{3}$ Aluno do programa de pós-graduação em Produção Vegetal, PGA/UEM, Maringá - PR.

Recebido pelo Conselho Editorial em: 14-11-2006

Aprovado pelo Conselho Editorial em: 22-6-2007
} 
A eficiência da utilização da água pela cultura do feijoeiro em relação ao rendimento obtido é um parâmetro de grande importância, sobretudo em locais onde a atividade de produção é diretamente determinada pela prática da irrigação, refletindo na rentabilidade e na eficiência da produção. Para a cultura do feijoeiro, o valor da eficiência de uso da água na produção de grãos com umidade de $10 \%$ é da ordem de 0,3 a $0,6 \mathrm{~kg} \mathrm{~m}^{-3}$, segundo DOORENBOS \& KASSAM (1979).

Para a avaliação dos efeitos dos diferentes níveis de uniformidade de irrigação sobre as diversas variáveis, conforme salientado por GONÇALVES (1997), torna-se necessário o tratamento estatístico dos dados, buscando descrever o comportamento de cada variável por meio de um conjunto de parâmetros, os quais descrevem a distribuição de freqüência dos dados.

Para associar à estimativa um nível de precisão, é necessário adotar uma medida que expresse a dispersão dos valores em torno da média. A variância ou o desvio-padrão dos dados descrevem essa dispersão. De acordo com WEBSTER \& OLIVIER (1990), o coeficiente de variação pode ser obtido pela relação entre o desvio-padrão e a média de um conjunto de dados, sendo útil para a comparação do nível de dispersão entre conjunto de dados, por ser adimensional.

Segundo LIBARDI et al. (1996), o desvio-padrão é recomendável como estatística de avaliação da variabilidade, pois possui a unidade original dos dados. Porém, a utilização do desviopadrão para a comparação de duas propriedades diferentes é inadequada, visto que as unidades são diferentes, necessitando-se, nesse caso, de medida relativa da variabilidade.

O coeficiente de variação foi utilizado como medida de variabilidade e serviu de base para os estudos de WARRICK \& NIELSEN (1980). Esses autores, estudando a variabilidade de diversas propriedades do solo, verificaram que, para algumas, a variabilidade expressa pelo coeficiente de variação pode ser inferior a $10 \%$, enquanto, para outras, pode superar $1.000 \%$. Classificaram a variabilidade em três níveis: baixo (abaixo de 12\%), médio (entre 12 e $80 \%$ ) e alto (acima de $80 \%$ ).

Para complementar a análise baseada nas medidas de posição e dispersão, uma análise descritiva dos dados no espaço é conveniente e pode ser realizada segundo métodos apresentados por ISAAKS \& SRIVASTAVA (1989). LIBARDI et al. (1996) apresentam ferramentas para a análise de dados distribuídos no espaço, tais como o diagrama de ramos e folhas. Outra ferramenta muito útil é o gráfico "box-plot”, conforme afirmam ISAAKS \& SRIVASTAVA (1989).

De acordo com MATEOS et al. (1997), a uniformidade da irrigação por aspersão é usualmente quantificada por coeficiente estatístico de uniformidade. Esses coeficientes, no entanto, mostram-se insuficientes para quantificar a influência da desuniformidade da irrigação sobre a produção da cultura, uma vez que os mesmos não levam em conta efeitos relacionados com as características do solo e, principalmente, da distribuição espacial da água aplicada.

Nas últimas décadas, a produção de cultura irrigada tem sido relacionada com a uniformidade de aplicação de água sobre a superfície do solo (FRIZZONE, 1998; MANTOVANI, 1993). No entanto, estudos mostram que a uniformidade da umidade do solo é, usualmente, maior que aquela medida sobre a superfície do mesmo (LI \& KAWANO, 1996; REZENDE et al., 2000) e que a planta pode responder favoravelmente a esse fato (GOHRING \& WALLENDER, 1987).

Assim, a análise do comportamento das variáveis adotadas, bem como das inter-relações entre elas, pode ser complementada com grandes benefícios à compreensão dos processos envolvidos, com o uso de técnicas de análise exploratória dos dados, principalmente levando-se em conta a distribuição dos dados no espaço.

Em conformidade com o exposto anteriormente, este trabalho teve como objetivo avaliar a produtividade do feijoeiro sob diferentes condições de uniformidade de água na superfície e na subsuperficie do solo, comparando a uniformidade de lâminas aplicadas e a uniformidade de umidades no solo. 


\section{MATERIAL E MÉTODOS}

$\mathrm{Na}$ área experimental de irrigação do Curso de Agronomia, da Universidade Estadual de Maringá - PR, localizada geograficamente a $23^{\circ} 25^{\prime}$ de latitude sul e a $51^{\circ} 57^{\prime}$ de longitude oeste, com altitude média de $542 \mathrm{~m}$, foi conduzido este trabalho. O solo da área é caracterizado como Nitossolo Vermelho distroférrico, com A moderado, textura argilosa. O relevo é suave ondulado, com declividade média de $8 \%$.

A análise granulométrica do solo presente na área experimental foi realizada com amostras obtidas na profundidade de 0 a $0,40 \mathrm{~m}$. Os valores médios obtidos para areia, silte, argila e densidade do solo são, respectivamente, $122,6 \mathrm{~g} \mathrm{~kg}^{-1} ; 120,6 \mathrm{~g} \mathrm{~kg}^{-1} ; 756,8 \mathrm{~g} \mathrm{~kg}^{-1}$, e 1,34 Mg m${ }^{-3}$.

O sistema de irrigação por aspersão utilizado para cada nível de uniformidade adotado foi composto de cinco linhas laterais adjacentes, com cinco aspersores por linha, conforme Figura 1, operando com pressão de serviço igual a 25 m.c.a e vazão média igual $2,36 \mathrm{~m}^{3} \mathrm{~h}^{-1}$. Trabalhou-se com quatro espaçamentos entre aspersores, com a finalidade de obter altos e baixos coeficientes de uniformidade de distribuição na superfície do solo, possibilitando a comparação com coeficientes de uniformidade de umidade abaixo da superfície. Os efeitos dos diferentes níveis de uniformidade sobre as variáveis de produção da cultura do feijoeiro também foram avaliados.

Os quatro espaçamentos entre aspersores foram denominados, neste trabalho, de cenários, sendo o cenário 1 correspondente ao espaçamento entre aspersores de $12 \times 12 \mathrm{~m}$ e os cenário 2; $3 \mathrm{e}$ 4 correspondentes, respectivamente, aos espaçamentos de 18 x 18 m, 18 x 24 m e 24 x 24 m.

As irrigações foram realizadas tendo como base a média das leituras de tensiômetros instalados em cada cenário, em quatro locais distintos, nas profundidades de 0,20 e 0,40 m, utilizando como limite os valores de umidade nas tensões de 0,05 MPa. Em todos os cenários, foi aplicada a mesma lâmina bruta de água, em todas as irrigações, igual a $12 \mathrm{~mm}$, possibilitando irrigar com maior freqüência e, conseqüentemente, maior número de irrigações para serem avaliadas.

Para a obtenção dos dados necessários aos cálculos dos parâmetros estatísticos que caracterizam a distribuição de água do sistema de irrigação, foram medidas as precipitações com o uso de pluviômetros dispostos em malhas de $3 \times 3 \mathrm{~m}$, nas áreas compreendidas entre os nove aspersores centrais, nos quatro cenários utilizados. Para cada um dos cenários, foram realizadas quatro amostragens. Em cada amostragem, considerou-se a área compreendida entre quatro aspersores. Em cada cenário, o número de quadrículas de $3 \times 3 \mathrm{~m}$ era diferente. Para os cenários 1; 2; 3 e 4, o número de quadrículas foi de 16;36; 48 e 64, respectivamente, conforme mostrado na Figura 1.

A determinação da uniformidade de distribuição da água abaixo da superfície do solo foi realizada a partir de medidas de umidade obtidas na profundidade de $0,30 \mathrm{~m}$, em dois pontos, situados a 0,30 m de cada pluviômetro, perfazendo duas repetições. As coletas das amostras de solo foram realizadas antes de se realizar cada uma das irrigações e também 24 horas após o seu final.

Neste trabalho, os coeficientes de uniformidade de irrigação foram obtidos para cada cenário, utilizando as lâminas coletadas. Determinaram-se, também, os coeficientes de uniformidade para as umidades do solo, na profundidade e no tempo já mencionados. Para cada irrigação, foram obtidos os coeficientes de uniformidade de Christiansen (CUC) e os coeficientes de uniformidade de distribuição (CUD).

A cultura do feijoeiro, cultivar IAPAR 57, foi escolhida para a condução do experimento, em função de sua alta resistência genética ao Vírus do Mosaico Dourado do Feijoeiro. Para a realização do estudo proposto, as variáveis foram avaliadas nas quatro amostragens, em cada cenário, nas mesmas épocas e utilizando-se sempre dos mesmos critérios, isto é, obtendo-se dados em cada quadrícula de $9 \mathrm{~m}^{2}$. As variáveis avaliadas foram número de vagens por planta (NV), número de grãos por vagem (NG), massa de 100 grãos (MC) e rendimento de grãos (RG). 


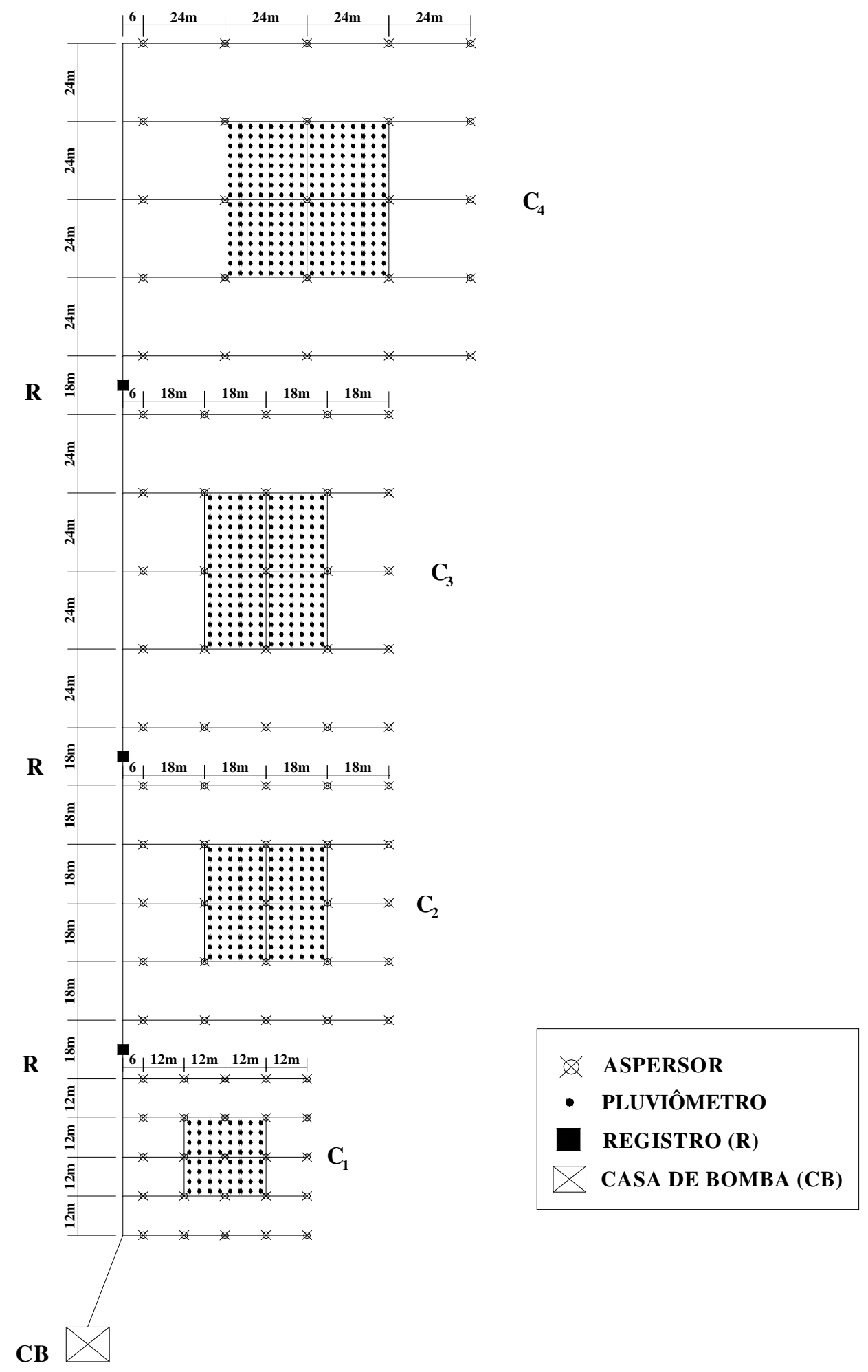

FIGURA1. Sistema de irrigação no campo, mostrando os quatro cenários $\left(\mathrm{C}_{1}, \mathrm{C}_{2}, \mathrm{C}_{3}\right.$ e $\left.\mathrm{C}_{4}\right)$.

Como salientado por MATEOS et al. (1997), os coeficientes de uniformidade de distribuição de água geralmente são insuficientes para expressar a influência da desuniformidade da irrigação sobre a produção da cultura, na medida em que não consideram a distribuição de água no espaço. Em vista desse fato, foi feita análise da distribuição dos dados de lâmina aplicada, de umidade do solo e de rendimento de grãos, no espaço. Para isso, foram usadas técnicas de análise exploratória que consistiram principalmente de um gráfico tipo "box-plot" simplificado, ou seja, onde são apresentados apenas os valores de mediana, máximo e mínimo das distribuições. 
A distribuição espacial dos valores das variáveis citadas foi estudada construindo-se os gráficos das estatísticas de ordem: mínimo, máximo e mediana, para os agrupamentos em linhas e em colunas, nos quatro cenários estudados. As linhas compreendem os coletores dispostos paralelamente às linhas laterais de irrigação, enquanto as colunas compreendem os coletores dispostos na direção ortogonal. Esse procedimento permitiu a análise do comportamento das variáveis no espaço, bem como das inter-relações entre os seus valores.

\section{RESULTADOS E DISCUSSÃO}

As condições climáticas durante os ensaios realizados, válidas para os quatro cenários, foram relativamente estáveis, não sendo possível detectar seus efeitos sobre os coeficientes de uniformidade obtidos. O valor médio da velocidade do vento foi igual a $0,62 \mathrm{~m} \mathrm{~s}^{-1}$, com coeficiente de variação (C.V.) de 8,0\%. A umidade relativa média foi de $58 \%$, com C.V. de 16,4\%, e a temperatura média foi de $20,6{ }^{\circ} \mathrm{C}$, com C.V. de $18,6 \%$. Quanto às precipitações ocorridas durante o ciclo da cultura (23-3 a 22-6), totalizaram 208,7 mm. Em decorrência do índice pluviométrico, somente foram realizadas 19 irrigações durante todo o ciclo da cultura, somando-se $228 \mathrm{~mm}$.

Na Tabela 1, são apresentados os valores médios, para 15 irrigações, dos coeficientes de uniformidade determinados acima (lâmina) e abaixo (umidade antes e depois de cada irrigação) da superfície do solo, para os quatro cenários. Nessa tabela, pode-se observar que a variação dos Coeficientes de Uniformidade de Christiansen para lâminas $\left(\mathrm{CUC}_{\mathrm{L}}\right)$, entre as irrigações, é baixa em todos os cenários, o que é expresso por valores de coeficientes de variação (C.V.) inferiores a $2 \%$. Isso mostra que o padrão de aplicação de água pouco se alterou com o passar do tempo, induzindo a comportamento semelhante da umidade do solo.

TABELA 1. Valores médios dos coeficientes de uniformidade determinados para lâmina $\left(\mathrm{CUC}_{\mathrm{L}}\right.$, $\left.\mathrm{CUD}_{\mathrm{L}}\right)$ e para umidade do solo antes $\left(\mathrm{CUC}_{\mathrm{UA}}, \mathrm{CUD}_{\mathrm{UA}}\right)$ e depois da irrigação $\left(\mathrm{CUC}_{\mathrm{UD}}, \mathrm{CUD}_{\mathrm{UD}}\right)$, média $(\overline{\mathrm{X}})$, desvio-padrão (s) e coeficiente de variação (C.V.) para os quatro cenários.

\begin{tabular}{lccccccccccccc}
\hline \multirow{2}{*}{ Coef. } & \multicolumn{3}{c}{ Cenário 1 } & \multicolumn{3}{c}{ Cenário 2 } & \multicolumn{3}{c}{ Cenário 3 } & \multicolumn{3}{c}{ Cenário 4 } \\
\cline { 2 - 13 } & $\bar{X}$ & s & C.V. & $\bar{X}$ & s & C.V. & $\bar{X}$ & S & C.V. & $\bar{X}$ & S & C.V. \\
\hline CUC $_{\mathrm{L}}$ & $94,1 \mathrm{a}$ & 0,69 & 0,7 & $85,6 \mathrm{~b}$ & 1,39 & 1,6 & $65,6 \mathrm{c}$ & 1,15 & 1,8 & $57,9 \mathrm{~d}$ & 0,53 & 0,9 \\
CUC $_{\mathrm{UA}}$ & $96,5 \mathrm{a}$ & 0,49 & 0,5 & $96,0 \mathrm{~b}$ & 0,53 & 0,6 & $95,8 \mathrm{c}$ & 0,68 & 0,7 & $96,2 \mathrm{~b}$ & 0,60 & 0,6 \\
CUC $_{\text {UD }}$ & $96,7 \mathrm{a}$ & 0,49 & 0,5 & $92,4 \mathrm{~b}$ & 0,65 & 0,7 & $85,3 \mathrm{c}$ & 0,77 & 0,9 & $75,6 \mathrm{~d}$ & 0,74 & 0,6 \\
CUD $_{\mathrm{L}}$ & $91,0 \mathrm{a}$ & 1,10 & 1,2 & $78,7 \mathrm{~b}$ & 2,18 & 2,8 & $46,2 \mathrm{c}$ & 1,82 & 3,9 & $23,6 \mathrm{~d}$ & 0,70 & 3,0 \\
CUD $_{\text {UA }}$ & $94,6 \mathrm{a}$ & 0,34 & 0,4 & $92,9 \mathrm{c}$ & 0,60 & 0,6 & $93,3 \mathrm{~b}$ & 0,49 & 0,5 & $92,6 \mathrm{c}$ & 0,68 & 0,7 \\
CUD $_{\text {UD }}$ & $94,7 \mathrm{a}$ & 0,81 & 0,9 & $87,9 \mathrm{~b}$ & 1,09 & 1,2 & $75,0 \mathrm{c}$ & 1,19 & 1,6 & $60,0 \mathrm{~d}$ & 0,42 & 0,7 \\
\hline
\end{tabular}

Médias seguidas da mesma letra na linha não diferem entre si, pelo teste de Tukey, a 5\% de probabilidade.

Os valores médios do Coeficiente de Uniformidade de Christiansen, obtidos para os dados de lâmina aplicada $\left(\mathrm{CUC}_{\mathrm{L}}\right)$, para os cenários 1 e 2, foram, respectivamente, 94,1 e 85,6, os quais são superiores ao valor mínimo aceitável citado em literatura (80\%), para o desempenho normal do sistema de aspersão. $\mathrm{O}$ mesmo não ocorre para os cenários 3 e 4 , cujos valores de $\mathrm{CUC}_{\mathrm{L}}$ são inferiores ao valor aceitável.

Os valores médios do Coeficiente de Uniformidade de Distribuição, obtidos para os dados de lâmina aplicada $\left(\mathrm{CUD}_{\mathrm{L}}\right)$, diminuíram com o aumento do espaçamento entre aspersores, de 91,0\% no cenário 1 , para $23,6 \%$ no cenário 4 , conforme pode ser observado na Tabela 1 . As diferenças entre os valores médios correspondentes aos cenários foram significativas pelo teste de Tukey, a $5 \%$ de probabilidade. Para o cenário 1, os valores médios de $\mathrm{CUC}_{\mathrm{L}}$ igual a $94,1 \%$ e $\mathrm{CUD}_{\mathrm{L}}$ igual a 91,0\% pouco diferem entre si, uma vez que a irrigação é realizada com alta uniformidade de lâmina aplicada. Para os demais cenários, a diferença entre os coeficientes cresce, como exemplo, no cenário $4, \mathrm{CUC}_{\mathrm{L}}$ é igual a 57,9\%, e $\mathrm{CUD}_{\mathrm{L}}$ igual a $23,6 \%$, evidenciando a desuniformidade da irrigação, bem como a maior sensibilidade do $\mathrm{CUD}_{\mathrm{L}}$ aos menores valores. Assim como para $\mathrm{CUC}_{\mathrm{L}}$, 
os valores de C.V. obtidos entre irrigações para o $\mathrm{CUD}_{\mathrm{L}}$ evidenciam a semelhança entre as 15 irrigações realizadas.

De acordo com a Tabela 1, todos os valores dos dois coeficientes de uniformidade determinados para umidade do solo, após a aplicação de água e antes da irrigação seguinte, foram superiores aos determinados para lâmina aplicada. Esse fato está relacionado com a redistribuição de água no solo e com o consumo diferenciado de água, isto é, a planta que está situada em local que não recebe água, consumirá menos que aquela que está próxima ao aspersor, onde a quantidade de água aplicada é maior. A redistribuição, ou seja, a tendência de a água movimentar-se de regiões mais úmidas para as de menor umidade, promove, após certo tempo, maior uniformidade da umidade, em relação à lâmina de água aplicada.

Também para os valores dos coeficientes calculados para as umidades após as irrigações, as diferenças entre cenários são estatisticamente significativas, a 5\% de probabilidade, para os dois coeficientes adotados. Nota-se que a variação em cada cenário, após as irrigações, foi pequena; para o $\mathrm{CUC}_{\mathrm{UD}}$, o coeficiente de variação foi inferior a $0,9 \%$ e, para o CUD $\mathrm{UD}_{\mathrm{D}}$, inferior a 1,6\%.

Os valores de CUC e de CUD calculados para a umidade do solo antes da irrigação (CUC UA $_{\mathrm{A}}$ e CUD UA $_{\text {I }}$ apresentam pequena variação entre cenários. Suas variações em cada cenário foram pequenas, o que é expresso por valores de coeficientes de variação (C.V.) inferiores a $0,7 \%$. Na Tabela 1, pode-se verificar que as diferenças de valores médios do $\mathrm{CUC}_{\mathrm{UA}}$ entre os cenários 2 e 4 não são significativas, mas diferem do cenário 3 , o qual também difere do cenário 1 . As mesmas variações podem ser verificadas para $C U D_{U A}$.

As estatísticas descritivas para os resultados das variáveis de produção, rendimento de grãos (RG), número de vagens por planta (NV), número de grãos por vagem (NG) e massa de 100 grãos (MC) são mostradas na Tabela 2, para os quatro cenários.

TABELA 2. Estatística descritiva para as variáveis de produção: rendimento de grãos em $\mathrm{kg} \mathrm{ha}^{-1}$ $(\mathrm{RG})$, número de vagens por planta $(\mathrm{NV})$, número de grãos por vagem $(\mathrm{NG})$ e massa de 100 grãos, em gramas (MC).

\begin{tabular}{|c|c|c|c|c|c|c|c|c|c|}
\hline \multirow{2}{*}{ Variáveis } & \multicolumn{9}{|c|}{ Estatística Descritiva } \\
\hline & $\mathrm{N}$ & Média & Mediana & Mín. & Máx. & $\mathrm{S}$ & C.V. & $\mathrm{d}$ & $\mathrm{d}^{*}$ \\
\hline RGc1 & \multirow{4}{*}{64} & $2.333,2 \mathrm{c}$ & $2.249,9$ & $1.503,6$ & $3.520,0$ & 429,4 & 18,4 & 0,13 & \multirow{4}{*}{0,17} \\
\hline NVc1 & & $9,2 \mathrm{~b}$ & 9,3 & 6,3 & 12,5 & 1,8 & 19,3 & 0,09 & \\
\hline NGc1 & & $5,8 \mathrm{~b}$ & 5,8 & 4,3 & 7,2 & 0,6 & 10,0 & 0,09 & \\
\hline MCc1 & & $21,6 \mathrm{~b}$ & 22,0 & 19,3 & 22,9 & 1,2 & 5,5 & 0,18 & \\
\hline $\mathrm{RGc2}$ & \multirow{4}{*}{144} & $2.759,1 \mathrm{a}$ & $2.877,9$ & $1.520,0$ & $3.521,0$ & 558,9 & 20,3 & 0,13 & \multirow{4}{*}{0,13} \\
\hline $\mathrm{NVc2}$ & & $10,0 \mathrm{a}$ & 9,0 & 5,8 & 15,3 & 2,2 & 21,9 & 0,18 & \\
\hline $\mathrm{NGc} 2$ & & $6,1 \mathrm{a}$ & 6,2 & 4,6 & 7,6 & 0,5 & 8,9 & 0,08 & \\
\hline $\mathrm{MCc} 2$ & & $22,2 \mathrm{a}$ & 22,4 & 19,4 & 23,9 & 1,1 & 4,8 & 0,09 & \\
\hline RGc3 & \multirow{4}{*}{192} & $2.422,8 \mathrm{~b}$ & $2.415,6$ & $1.503,6$ & $3.655,4$ & 525,0 & 21,7 & 0,08 & \multirow{4}{*}{0,10} \\
\hline $\mathrm{NVc3}$ & & $9,6 \mathrm{ab}$ & 9,3 & 5,8 & 15,3 & 2,0 & 20,8 & 0,12 & \\
\hline $\mathrm{NGc3}$ & & $5,6 \mathrm{~b}$ & 5,8 & 3,0 & 7,2 & 0,8 & 14,8 & 0,16 & \\
\hline $\mathrm{MCc} 3$ & & $21,8 \mathrm{~b}$ & 22,1 & 16,0 & 23,9 & 1,9 & 8,6 & 0,2 & \\
\hline $\mathrm{RGc4}$ & \multirow{4}{*}{256} & $1.501,5 \mathrm{~d}$ & $1.466,8$ & 835,4 & $2.315,6$ & $3.06,7$ & 20,4 & 0,12 & \multirow{4}{*}{0,08} \\
\hline $\mathrm{NVc4}$ & & $7,9 \mathrm{c}$ & 7,8 & 2,0 & 13,0 & 2,1 & 26,0 & 0,11 & \\
\hline NGc4 & & $4,6 \mathrm{c}$ & 4,6 & 2,0 & 5,8 & 0,7 & 16,3 & 0,18 & \\
\hline $\mathrm{MCc} 4$ & & $20,1 \mathrm{c}$ & 20,5 & 15,2 & 22,5 & 1,4 & 7,1 & 0,15 & \\
\hline
\end{tabular}

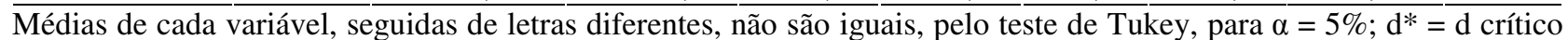
pelo teste de Kolmogorov-Smirnov, para $\alpha=5 \%$.

O rendimento de grãos da cultura foi influenciado pela qualidade da irrigação realizada. Os diferentes cenários originaram produções que diferem entre si, conforme apresentado na Tabela 2. A maior produtividade, no entanto, foi obtida no cenário $2\left(2.759,1 \mathrm{~kg} \mathrm{ha}^{-1}\right)$, correspondente ao 
espaçamento de 18 x 18m. A produtividade obtida no cenário com maior uniformidade (cenário 1) foi maior que a obtida no cenário 4 apenas, demonstrando que a maior uniformidade não implicou máxima produtividade.

O número de vagens por planta seguiu o mesmo comportamento da variável rendimento de grãos. O maior valor foi obtido no cenário $2(10,0)$, e o valor obtido no cenário $1(9,2)$ foi maior que o do cenário $4(7,9)$ apenas. Para essa variável, no entanto, o cenário 3 deu origem a um valor que não difere estatisticamente daqueles obtidos para os cenários 1 e 2, expressando a menor sensibilidade dessa variável às diferentes condições de irrigação adotadas.

O número de grãos por vagem apresentou comportamento semelhante ao das outras variáveis. O maior valor foi obtido para o cenário $2(\mathrm{NGc} 2=6,1)$ e o menor para o cenário 4 (NGc4 = 4,6). Os cenários 1 e 3 deram origem a valores que não diferiram estatisticamente entre si. O mesmo ocorreu com a massa de 100 grãos, mostrando que a irrigação mais favorável à cultura não foi a realizada com maior uniformidade, mas aquela referente ao espaçamento de $18 \times 18 \mathrm{~m}$, a qual pode ser caracterizada como de alta uniformidade, segundo os critérios usuais (CUC $=85,6 \%$ para lâmina aplicada).

O rendimento médio de grãos no cenário 2 (RGc2) foi de $2.759 \mathrm{~kg} \mathrm{ha}^{-1}$, valor que pode ser considerado elevado, mesmo em condições de irrigação, para a latitude em questão (FANCELLI \& DOURADO-NETO, 1997). Para os cenários 3 e 1, os rendimentos médios foram de 2.423 e $2.333 \mathrm{~kg} \mathrm{ha}^{-1}$, respectivamente, revelando, também, alta produtividade. Para o cenário 4, o rendimento médio de $1.500 \mathrm{~kg} \mathrm{ha}^{-1}$ revela a redução de produtividade associada à redução da uniformidade da irrigação, embora a lâmina média tenha sido constante entre cenários. Isso torna o espaçamento referente ao cenário 3 uma condição conveniente, uma vez que substancial redução de equipamento necessário promoveu pequena redução de produtividade.

A distribuição espacial dos valores de lâmina coletada, de umidade e de rendimento de grãos da cultura do feijoeiro foi analisada por meio de técnicas da análise exploratória de dados distribuídos no espaço. Em cada cenário, foram tomados os valores de mediana, máximo e mínimo para os dados agrupados em colunas e em linhas, os quais são apresentados nas Figuras 2; 3 e 4.

A distribuição espacial dos valores de lâmina coletada, nos quatro cenários, pode ser avaliada observando-se os gráficos apresentados na Figura 2. No cenário 1, a alta uniformidade de aplicação de água fica evidenciada pela semelhança entre as medianas de todas as colunas e linhas e pela pequena dispersão em torno desse parâmetro, para todos os agrupamentos de dados (linhas e colunas), caracterizada por coeficientes de variação entre $1,0 \%$ e $6,7 \%$.

Para o cenário 2, o espaçamento quadrado entre aspersores de 18 x $18 \mathrm{~m}$ fez com que as distribuições dos dados agrupados em colunas e em linhas fossem semelhantes. A pequena variação dos valores de mediana em torno do valor médio de $11,9 \mathrm{~mm}$, para todos os agrupamentos, pode ser visualizada na Figura 2. Em cada agrupamento, os valores de C.V. são maiores para o cenário 1, e variam mais entre agrupamentos, assumindo valores entre 4,2 e 25,1\%.

Embora os C.V. revelem maiores dispersões em relação ao cenário 1, os valores máximos pouco superam $15 \mathrm{~mm}$, e os valores mínimos atingem valores pouco abaixo de $10 \mathrm{~mm}$, revelando alta uniformidade da irrigação no cenário 2.

No cenário 3, com o espaçamento retangular entre aspersores de 18 x $24 \mathrm{~m}$, a dispersão dos valores de mediana em torno do valor médio igual a $11,7 \mathrm{~mm}$, nas colunas, é pequena, semelhante ao que ocorre para o cenário 2. Os C.V., no entanto, assumem valores bem mais elevados, da ordem de até 71,3\%, evidenciando a maior irregularidade das lâminas no espaço.

Nesse cenário 3, a distribuição das medianas dos agrupamentos em linhas assume tendência semelhante à do cenário 4. Em função do espaçamento de $24 \mathrm{~m}$, grande para o aspersor utilizado, cujo diâmetro molhado foi de $25 \mathrm{~m}$, os valores de mediana são consideravelmente menores na região central entre aspersores e maiores próximo a eles. 

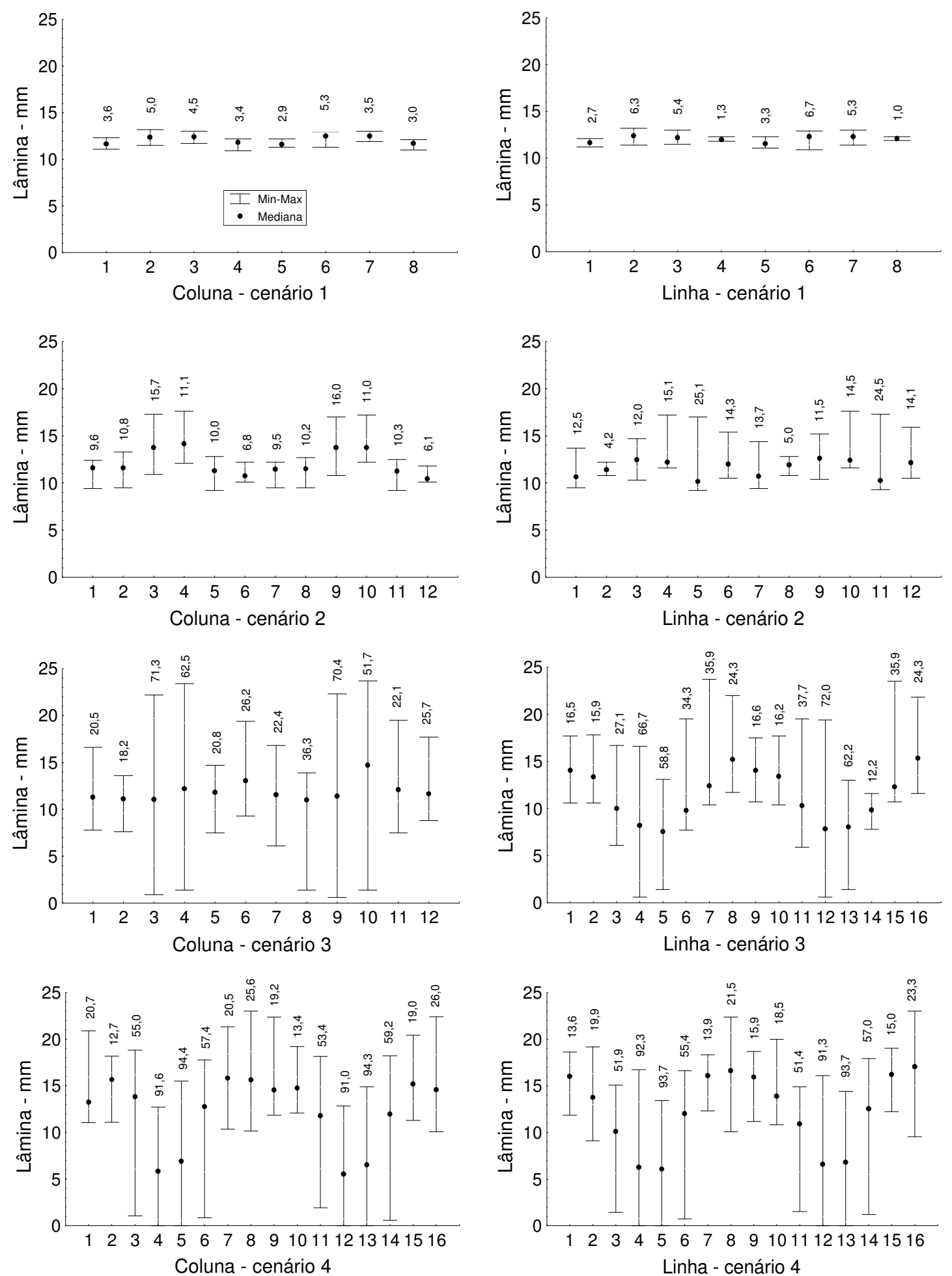

FIGURA 2. Valores mínimo, máximo, mediana e de coeficiente de variação para dados de lâmina coletada, nas colunas e nas linhas, para os cenários $1 ; 2 ; 3$ e 4.

No cenário 3 e, principalmente, no 4, os valores mínimos de lâmina coletada tendem a zero, na região central da área irrigada por quatro aspersores. Para obter lâmina média aproximadamente constante em cada cenário, da ordem de $12 \mathrm{~mm}$, é necessário que o tempo de irrigação cresça com o espaçamento entre aspersores. Isso faz com que os valores máximos nos agrupamentos cresçam, atingindo valores acima de $20 \mathrm{~mm}$.

Na Figura 3, são mostradas as distribuições espaciais dos valores de umidade do solo após as irrigações, agrupadas em colunas e linhas, nos quatro cenários. A alta uniformidade de irrigação é expressa por uniformidade ainda mais elevada dos valores de umidade no cenário 1 . A pequena dispersão dos valores de mediana em torno do valor médio pode ser visualizada na Figura 3 . A 
dispersão dos valores em torno da mediana, em cada agrupamento, está associada ao C.V. entre $0,7 \%$ e $2,1 \%$, evidenciando elevada uniformidade, maior que a identificada para a lâmina de irrigação (Figura 2), o que está associada à redistribuição da água no solo.
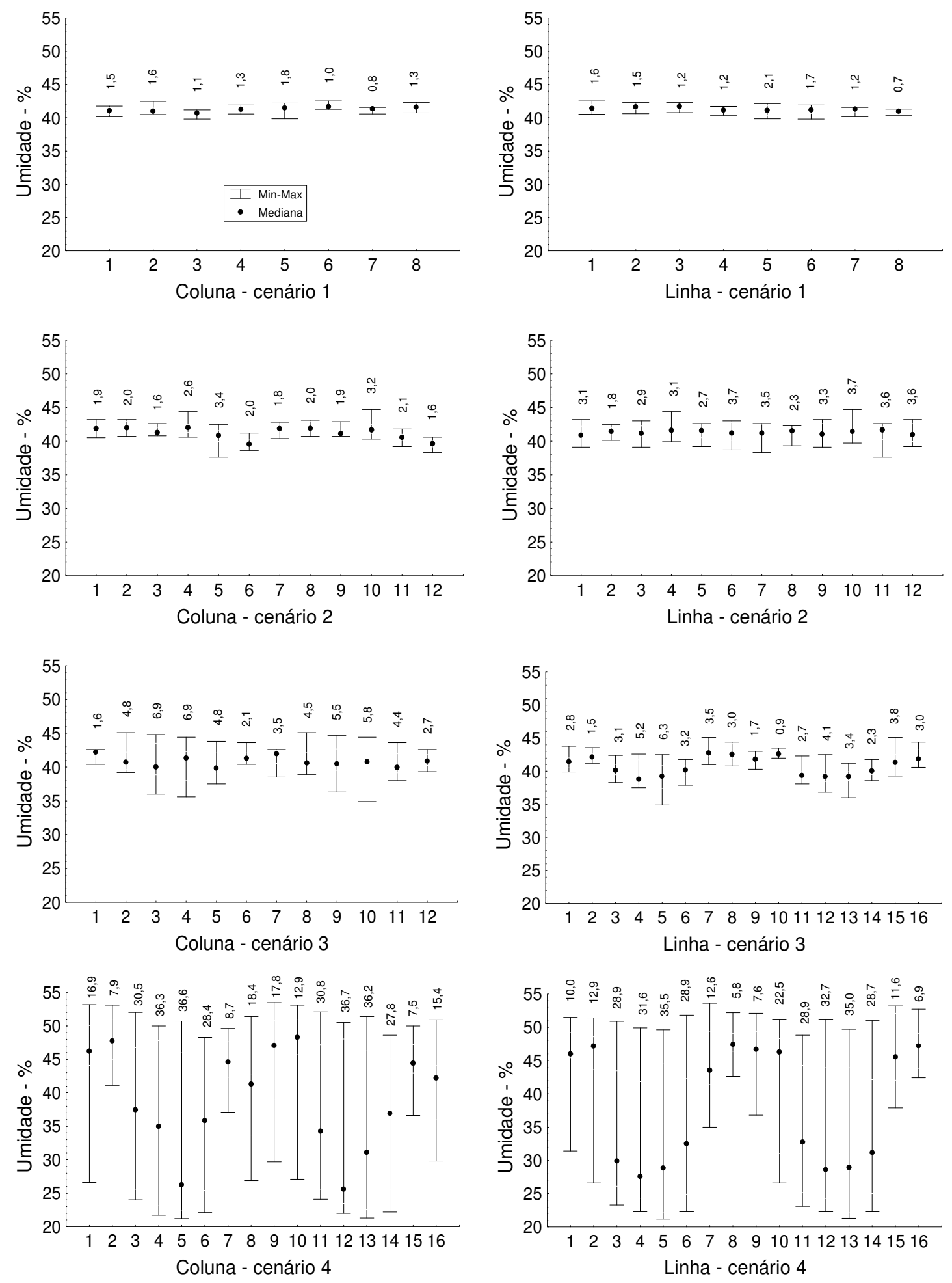

FIGURA 3. Valores mínimo, máximo, mediana e de coeficiente de variação para dados de umidade do solo após as irrigações, nas colunas e nas linhas, para os cenários 1; 2; 3 e 4.

No cenário 2, a redistribuição da água no solo é também evidente, contribuindo para uniformidade da umidade expressa por valores de C.V. entre $1,6 \%$ e 3,7\%, para os agrupamentos em colunas e em linhas. Os valores de mediana variaram em torno de 41,3\%, valor médio. Comparando-se as Figuras 2 e 3 para esse cenário, constata-se que a redistribuição de água no solo reduz substancialmente a dispersão em torno da mediana, reduzindo a amplitude de variação dos dados de umidade em relação aos de lâmina de irrigação. 
No cenário 3, os agrupamentos em colunas apresentam valores de mediana que variam pouco em torno do valor médio, da ordem de $41,2 \%$, de modo semelhante ao que ocorreu para as lâminas. Nos agrupamentos em linha, com os aspersores espaçados de $24 \mathrm{~m}$, pode-se verificar que, assim como o ocorrido para lâminas, os valores de mediana são menores na região entre aspersores e maiores na região próximo aos aspersores. Para esse espaçamento, grande para o aspersor utilizado, a redistribuição de umidade não é mais suficiente para manter os valores de mediana uniformes em toda a área irrigada, como nos espaçamento anteriores. Esse fato fica evidenciado ao se compararem os cenários 1 e 2 e os agrupamentos em colunas para o cenário 3 , nos quais os valores de mediana pouco variam, com o cenário 4, no qual a variação entre agrupamentos é muito grande.

O cenário 4 apresenta-se com grande diferença em relação aos demais, em termos de distribuição espacial de água no solo. $\mathrm{O}$ fato de algumas regiões centrais das quadrículas entre quatro aspersores não terem recebido água, faz com que, apenas neste cenário, a redistribuição de água no solo não seja capaz de uniformizar os valores de mediana dos agrupamentos. Isso revela que pontos mais distantes dos aspersores têm valores de umidade da ordem de $20 \%$ (valores mínimos), enquanto pontos mais próximos dos aspersores apresentam valores da ordem de $50 \%$ ou mais (valores máximos). Esse fato mostra a forte dispersão em torno do valor central dentro dos agrupamentos, o que é expresso por valores de C.V. entre 5,8 e 36,7\%.

Com o propósito de avaliar as relações entre as distribuições espaciais de lâmina e de umidade e distribuição de valores de rendimento de grãos, foram construídos os gráficos da Figura 4. Para o cenário 1, verifica-se que os agrupamentos em colunas e linhas apresentaram valores de mediana com dispersão em torno de valor médio, igual a $2.249,9 \mathrm{~kg} \mathrm{ha}^{-1}$, superior ao verificado para as lâminas e, principalmente, para umidades.

Importante é observar que essa variabilidade que se manifestou no ambiente onde a uniformidade da umidade do solo é quase absoluta e, portanto, pode ser atribuída aos fatores nãocontrolados, foi expressivamente maior que a variabilidade da umidade do solo nos cenários 1; 2 e 3. Apenas no cenário 4 é que os valores de C.V. para os agrupamentos de dados chegam a superar esses valores de C.V. para rendimento de grãos no cenário 1 .

Para os cenários 1; 2 e 3, verifica-se que a redistribuição suavizou a distribuição de lâminas, tornando a distribuição espacial de umidade uniforme o bastante para que a variabilidade do rendimento de grãos fosse maior que a decorrente da variação da irrigação realizada. Isso permite a obtenção do resultado de maiores valores de média e de mediana do rendimento de grãos no cenário 2, seguido dos valores no cenário 3, ambos maiores que o cenário 1; nesse, os valores máximos da maior parte dos agrupamentos ficou abaixo de $3.000 \mathrm{~kg} \mathrm{ha}^{-1}$. No cenário $3 \mathrm{e}$, principalmente, no cenário 2, alguns valores máximos ficaram acima de $3.500 \mathrm{~kg} \mathrm{ha}^{-1} \mathrm{e}$, na maioria dos agrupamentos, ficaram acima de $3.000 \mathrm{~kg} \mathrm{ha}^{-1}$.

Os valores de rendimento de grãos no cenário 4 apresentam mediana dos agrupamentos expressivamente menores que os demais cenários (aproximadamente $50 \%$ do obtido no cenário 2). O grande espaçamento adotado faz com que, nesse cenário, as medianas dos agrupamentos assumam distribuição no espaço que acompanha a distribuição dos valores correspondentes de lâmina e de umidade do solo. Nesse cenário, a cultura respondeu à variação da umidade do solo, decorrente da variação espacial da lâmina de irrigação, em termos das medidas de tendência central dos dados nos agrupamentos (mediana e média).

Entretanto, os valores máximos de umidade nos diversos agrupamentos do cenário 4 foram relativamente uniformes (bem mais uniformes que os valores de mediana), entre $50 \%$ e $55 \%$. Esses valores elevados estão associados a valores máximos de rendimento de grãos entre $2.000 \mathrm{e}$ $2.500 \mathrm{~kg} \mathrm{ha}^{-1}$, consideravelmente menores que os valores máximos obtidos, principalmente nos cenários 2 e 3. Mais uma vez, a variabilidade no rendimento de grãos, em resposta à variabilidade de irrigação, não foi maior que no cenário 1 , de uniformidade quase absoluta. A irrigação influenciou no valor da mediana dos valores de rendimento de grãos de cada agrupamento, mas não afetou a dispersão dos valores em torno dessa posição central. 

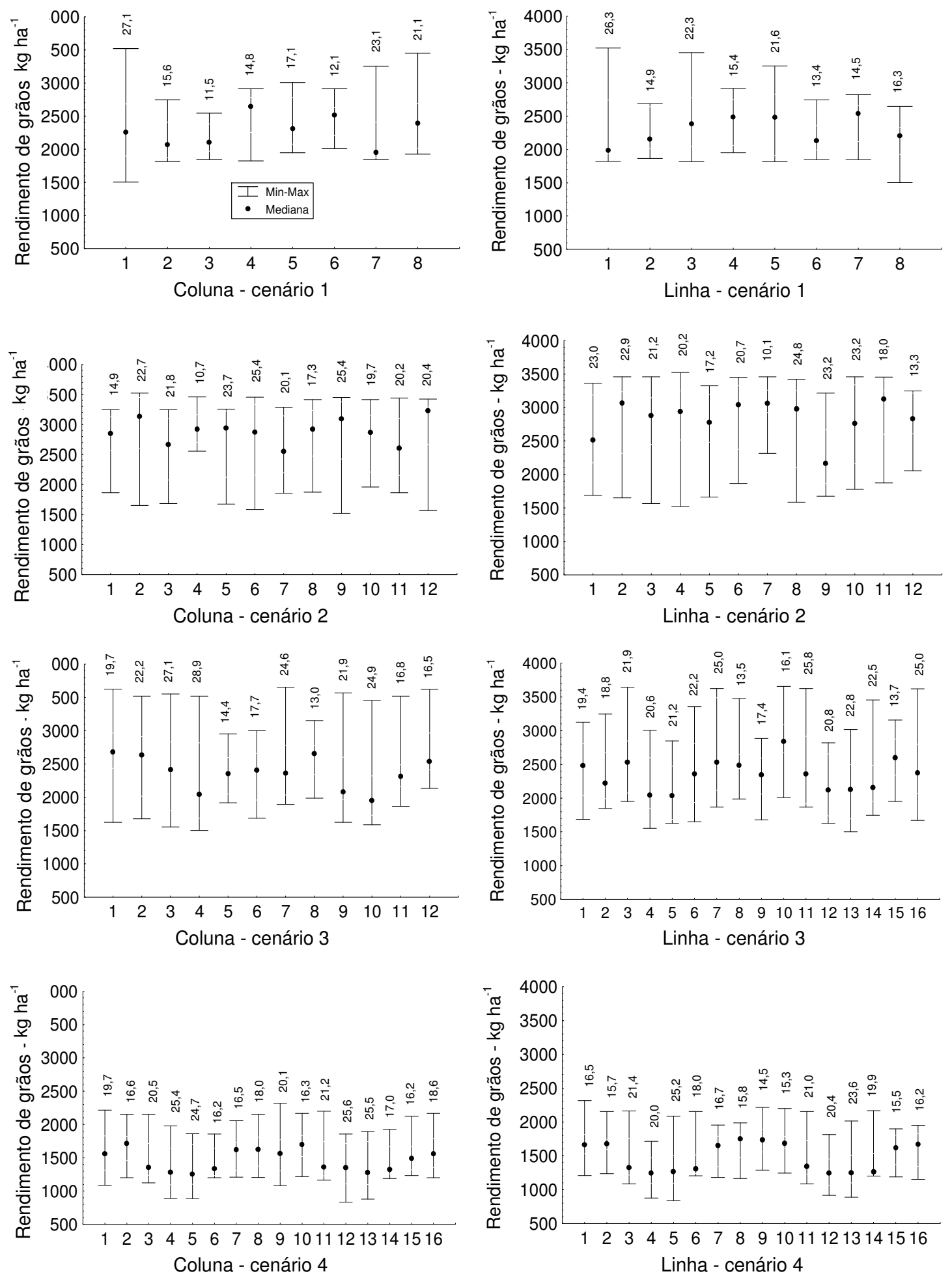

FIGURA 4. Valores mínimo, máximo, mediana e de coeficiente de variação para dados de rendimentos de grãos, nas colunas e nas linhas, para os cenários $1 ; 2 ; 3$ e 4.

Paralelamente, os elevados valores de lâmina de irrigação aplicados em algumas posições no cenário 4 afetaram de forma negativa a produtividade da cultura nessas regiões, provavelmente, por promover maior lixiviação de nutrientes e inadequada aeração do solo, acarretando redução dos valores máximos de rendimento de grãos. Por outro lado, os valores nulos de lâminas em algumas áreas foram compensados pela redistribuição de água no solo e, em parte, pelas chuvas, levando a cultura a produzir uma quantidade que, embora menor que os valores mínimos de outros cenários, foi ainda apreciável (Figura 4). 
Em decorrência desses fatos, a amplitude dos valores nos agrupamentos do cenário 4 foi ligeiramente menor que nos demais cenários. Associada aos menores valores médios, deram origem a valores de C.V. semelhantes aos obtidos nos demais cenários.

\section{CONCLUSÕES}

A uniformidade da umidade do solo, embora dependente da uniformidade da lâmina aplicada, foi consideravelmente maior que essa, nas condições estudadas.

A qualidade da irrigação interferiu na uniformidade da umidade do solo e influenciou nas variáveis de produção da cultura de feijão.

Uniformidade máxima de aplicação de água na superfície do solo não implicou maior rendimento de grãos.

\section{REFERÊNCIAS}

DOORENBOS, J.; KASSAM, A.H. Efectos del água sobre el rendimento de los cultivos. Rome: FAO, 1979. (Riego y Drenaje, 33).

FANCELLI, A.L.; DOURADO NETO, D. Ecofisiologia e fenologia do feijoeiro. In: FANCELLI, A.L; DOURADO NETO, D. (Coord.) Tecnologia da produção do feijão irrigado. Piracicaba: ESALQ, Departamento de Agricultura, 1997. p.100-20.

FRIZZONE, J.A. Uniformidade e eficiência da irrigação. Piracicaba: ESALQ/Departamento de Engenharia Rural, 1998. 40 p.

GOHRING, R.T.; WALLENDER, W.W. Economics of sprinkler irrigation systems. Transactions of the ASAE, St. Joseph, v.30, n.4, p.1.083-9, 1987.

GONÇALVES, A.C.A. Variabilidade espacial de propriedades físicas do solo para fins de manejo da irrigação. 1997. Tese (Doutorado em Irrigação e Drenagem) - Escola Superior de Agricultura "Luiz de Queiroz", Universidade de São Paulo, Piracicaba, 1997.

ISAAKS, E.H.; SRIVASTAVA, R.M. An introduction to applied geostatistics. New York: Oxford University Press, 1989.

LIBARDI, P.L.; MANFRON, P.A.; MORAES, S.O. de; TUON, R.L. Variabilidade da umidade gravimétrica de um solo hidromórfico. Revista Brasileira de Ciência do Solo, Viçosa, v.20, p.1-12, 1996.

LI, J.; KAWANO, H. The areal distribution of soil moisture under sprinkler irrigation. Agricultural Water Management, Amsterdam, v.32, n.1, p.29-36, 1996.

MANTOVANI, E.C. Desarrolo y evaluacion de modelos para el manejo del riego: estimación de la evapotranspiracion y efectos de la uniformidad de aplicacion del riego sobre a produccion de los cultivos. 1993. Tesis (Doctor) - Escuela Tecnica Superior de Ingenieros Agronomos, Universidade de Córdoba, Córdoba, 1993.

MATEOS, L.; MANTOVANI, E.C.; VILLALOBOS, F.J. Cotton response to non-uniformity of conventional sprinkler irrigation. Irrigation Science, Berlim, v.17, n.1, p.47-52, 1997.

REZENDE, R.; GONÇALVES, A.C.A.; FRIZZONE, J.A.; FARIA, M. A. Uniformidade da irrigação por aspersão acima e abaixo da superfície do solo e seu efeito sobre a produção da cultura do feijoeiro. In.: BALBUENA, R.H.; BENEZ, S.H.; JORAJURIA, C.D. (Eds.). Avances en Ingeniería Agrícola. Buenos Aires: Facultad Agronomia, 2000. p.290-5.

WARRICK, A.W.; NIELSEN, D.R. Spatial variability of soil physical properties in the field. In: HILLEL, D. (Ed.). Application of soil physics. New York: Academic Press, 1980. p.319-44.

WEBSTER, R.; OLIVIER, M.A. Statistical methods in soil and land resource survey. Oxford: University Press, 1990. 316 p. 